\title{
Periodic boundary value problems for second order functional differential equations
}

\author{
Weibing Wang · Jianhua Shen · Juan J. Nieto
}

Received: 18 January 2010 / Published online: 16 April 2010

(C) The Author(s) 2010. This article is published with open access at Springerlink.com

\begin{abstract}
This paper deals with a periodic boundary value problem for a second order functional differential equation. We obtain the existence of extreme solutions under new concept of upper and lower solutions. Also, a mistake in a recent paper (Ding et al. in J. Math. Anal. Appl. 298:341-351, 2004) is corrected.
\end{abstract}

Keywords Periodic boundary value problems - Upper and lower solutions · Comparison theorem $\cdot$ Functional differential equations

Mathematics Subject Classification (2000) 34B15 · 34B16

\section{Introduction}

It is well known that the monotone iterative technique offers an approach for obtaining approximate solutions of nonlinear differential equations. There also exist much literature devoted to the applications of this technique to boundary value problems of functional differential equations, see [1, 7-16] and the references therein. In [2, 3], J.J. Nieto and R. Rodriguez-Lopez introduced a new concept of lower and upper so-

\footnotetext{
W. Wang $(\bowtie)$

Department of Mathematics, Hunan University of Science and Technology, Xiangtan, Hunan 411201, China

e-mail:wwbhnnuorsc@yahoo.com.cn

J. Shen

Department of Mathematics, Hangzhou Normal University, Hangzhou, Zhejiang 310036, China

J.J. Nieto

Departamento de Análisis Matemático, Facultad de Matemáticas,

Universidad de Santiago de Compostela, 15782 Santiago de Compostela, Spain
} 
lutions, and considered the periodic boundary value problems for the following first order functional differential equation

$$
\left\{\begin{array}{l}
u^{\prime}(t)=g(t, u(t), u(\theta(t))), \quad t \in[0, T], \\
u(0)=u(T) .
\end{array}\right.
$$

A similar method has already succeeded in employing to nonlinear impulsive integrodifferential equations [4] and impulsive functional differential equations [5]. In this paper, we consider the following second order functional differential equation

$$
\left\{\begin{array}{l}
-u^{\prime \prime}(t)=f(t, u(t), u(\theta(t))), \quad t \in J=[0, T], \\
u(0)=u(T), \quad u^{\prime}(0)=u^{\prime}(T),
\end{array}\right.
$$

where $f \in C\left(J \times R^{2}, R\right), 0 \leq \theta(t) \leq t, t \in J$.

In a recent paper [6], authors had extended the concept of lower and upper solutions for (1.1). By using the method of upper and lower solutions and monotone iterative technique, they obtained the existence of extreme solutions for the boundary value problem (1.1). However, the results of [6] do not hold. In order to show this, we list the main comparison theorems.

Let $E^{*}=C(J, R) \cap C^{2}(J, R), c^{*}(t)=\min \{t, T-t\}, t \in J$ and $M \in R, N \in R$. The authors gave the following definition in Sect. 2 of [6] (see Definition 2.1 and Definition 2.2 in [6]).

Definition 1 A function $\alpha \in E^{*}$ is called a lower solution of the boundary value problem (1.1) if

$$
\left\{\begin{array}{l}
-\alpha^{\prime \prime}(t) \leq f(t, \alpha(t), \alpha(\theta(t)))-a(t), \quad t \in J, \\
\alpha(0)=\alpha(T),
\end{array}\right.
$$

where

$$
a(t)= \begin{cases}0, & \alpha^{\prime}(0) \geq \alpha^{\prime}(T), \\ \left(M c^{*}(t)+N c^{*}(\theta(t))\right)\left(\alpha^{\prime}(T)-\alpha^{\prime}(0)\right), & \alpha^{\prime}(0)<\alpha^{\prime}(T) .\end{cases}
$$

Definition 2 A function $\beta \in E^{*}$ is called an upper solution of the boundary value problem (1.1) if

$$
\left\{\begin{array}{l}
-\beta^{\prime \prime}(t) \geq f(t, \beta(t), \beta(\theta(t)))+b(t), \quad t \in J \\
\beta(0)=\beta(T)
\end{array}\right.
$$

where

$$
b(t)= \begin{cases}0, & \beta^{\prime}(0) \leq \beta^{\prime}(T), \\ \left(M c^{*}(t)+N c^{*}(\theta(t))\right)\left[\beta^{\prime}(0)-\beta^{\prime}(T)\right], & \beta^{\prime}(0)>\beta^{\prime}(T) .\end{cases}
$$


Consequently, they established the following two comparison results (Lemma 2.1 and Lemma 2.2 in [6]) corresponding to the above new concept of lower and upper solutions.

Lemma 1.1 Assume that $u \in E^{*}$ satisfies

$$
\left\{\begin{array}{l}
-u^{\prime \prime}(t)+M u(t)+N u(\theta(t)) \leq 0, \quad t \in J \\
u(0)=u(T), \quad u^{\prime}(0) \geq u^{\prime}(T),
\end{array}\right.
$$

where constants $M>0, N \geq 0$ satisfy

$$
T^{2}(M+N) \leq 1
$$

Then $u(t) \leq 0$ for all $t \in J$.

Lemma 1.2 Assume that $u \in E^{*}$ satisfies

$$
\left\{\begin{array}{l}
-u^{\prime \prime}(t)+M u(t)+N u(\theta(t))+\left[M c^{*}(t)+N c^{*}(\theta(t))\right]\left(u^{\prime}(T)-u^{\prime}(0)\right) \leq 0, \\
\quad t \in J, \\
u(0)=u(T), \quad u^{\prime}(0)<u^{\prime}(T),
\end{array}\right.
$$

where constants $M>0, N \geq 0$ satisfy (1.2). Then $u(t) \leq 0$ for all $t \in J$.

Remark 1 In [6], authors used Lemma 1.1 to prove Lemma 1. 2. However, Lemma 1.2 is false. For example, let

$$
u_{0}(t)=t(t-T)+1, \quad M=N=10^{-4} \min \left\{T^{-2}, 10^{-4}\right\},
$$

then $u_{0}(0)=u_{0}(T)=1, u_{0}^{\prime}(T)-u_{0}^{\prime}(0)=2 T$ and

$$
\begin{aligned}
& -u_{0}^{\prime \prime}(t)+M u_{0}(t)+N u_{0}(\theta(t))+\left[M c^{*}(t)+N c^{*}(\theta(t))\right]\left(u_{0}^{\prime}(T)-u_{0}^{\prime}(0)\right) \\
& \leq-2+M+N+2 \times 10^{-4}<0 .
\end{aligned}
$$

In fact, Lemma 1.1 cannot be used in the proof of Lemma 1.2 as in [6], because the authors put

$$
y(t)=u(t)+c^{*}(t)\left(u^{\prime}(T)-u^{\prime}(0)\right) .
$$

Obviously, $y^{\prime}(t)$ does not exist at $t=T / 2$ and so $y \bar{\in} E^{*}$. Thus the main theorem of [6] does not hold since Lemma 1.2 was used repeatedly in [6].

The aim of this work is to prefect the method of [6]. We use a new technique in the proof so that the required conditions to satisfy the main theorem are minimized. The rest of the paper is organized as follows. In Sect. 2, we establish three comparison principles. In Sect. 3, we give a proof for the existence theorem related to a linear problem associated to (1.1). In Sect. 4, we first introduce new concept of lower and upper solutions, and by using the method of upper and lower solutions and monotone iterative technique, we obtain the existence of extreme solutions for (1.1). 


\section{Comparison results}

Put

$$
E=C^{2}(J, R), \quad c(t)=\frac{T}{2 \pi} \sin \frac{\pi}{T} t .
$$

We now present main results of this section.

Lemma 2.1 Assume that $u \in E$ satisfies

$$
\left\{\begin{array}{l}
-u^{\prime \prime}(t)+M u(t)+N u(\theta(t)) \leq 0, \quad t \in J, \\
u(0)=u(T), \quad u^{\prime}(0) \geq u^{\prime}(T),
\end{array}\right.
$$

where constants $M>0, N \geq 0$ satisfy

$$
T^{2}(M+N) \leq 2 .
$$

Then $u(t) \leq 0$ for all $t \in J$.

Proof Suppose, to the contrary, that $u(t)>0$ for some $t \in J$. We consider the following two cases.

Case $1 u(t) \geq 0, u(t) \neq 0$ on $J$. It is easy to obtain that $u^{\prime \prime}(t) \geq 0$ on $J$. Thus $u(t) \equiv K>0$ since $u^{\prime}(0) \geq u^{\prime}(T)$. Consequently, we obtain

$$
(M+N) K=-u^{\prime \prime}(t)+M u(t)+N u(\theta(t)) \leq 0,
$$

which contradicts $K>0$.

Case 2 There exist $t_{1}, t_{2} \in J$ such that $u\left(t_{1}\right)>0$ and $u\left(t_{2}\right)<0$. Hence, two cases are possible.

Subcase $2.1 u(0)=u(T)<0$. There exists a $t_{3} \in(0, T)$ such that

$$
u\left(t_{3}\right)=\max _{t \in J} u(t)>0, \quad u^{\prime}\left(t_{3}\right)=0 .
$$

Let $u\left(t_{*}\right)=\min _{t \in\left[0, t_{3}\right)} u(t)<0$. Then

$$
u^{\prime \prime}(t) \geq(M+N) u\left(t_{*}\right), \quad t \in\left[0, t_{3}\right) .
$$

Integrating the above inequality from $s\left(t_{*} \leq s \leq t_{3}\right)$ to $t_{3}$, we obtain

$$
-u^{\prime}(s) \geq\left(t_{3}-s\right)(M+N) u\left(t_{*}\right), \quad t_{*} \leq s \leq t_{3},
$$

and then integrate from $t_{*}$ to $t_{3}$ to obtain

$$
\begin{aligned}
-u\left(t_{*}\right) & <u\left(t_{3}\right)-u\left(t_{*}\right) \leq \int_{t_{*}}^{t_{3}}\left(s-t_{3}\right)(M+N) u\left(t_{*}\right) d s \\
& \leq-\frac{M+N}{2} u\left(t_{*}\right)\left(t_{3}-t_{*}\right)^{2} \leq-\frac{M+N}{2} u\left(t_{*}\right) T^{2},
\end{aligned}
$$


which implies that $1<\frac{T^{2}}{2}(M+N)$. This is a contradiction.

Subcase $2.2 u(0)=u(T) \geq 0$. There exists a $t_{3} \in J$ such that

$$
u\left(t_{3}\right)=\max _{t \in J} u(t)>0 .
$$

If $t_{3} \in(0, T)$, then $u^{\prime}\left(t_{3}\right)=0$. If $t_{3}=0$ or $t_{3}=T$, then $u^{\prime}(0) \leq 0 \leq u^{\prime}(T)$. So $u^{\prime}(0)=$ $u^{\prime}(T)=0$.

Let $u\left(t_{*}\right)=\min _{t \in(0, T)} u(t)<0$. Then

$$
u^{\prime \prime}(t) \geq(M+N) u\left(t_{*}\right), \quad t \in J .
$$

When $t_{*}<t_{3}$, same as subcase 2.1 , we obtain that $1<T^{2}(M+N) / 2$. When $t_{*}>t_{3}$, integrating the inequality (2.2) from $t_{3}$ to $s\left(t_{3} \leq s \leq t_{*}\right)$, we obtain

$$
u^{\prime}(s) \geq\left(s-t_{3}\right)(M+N) u\left(t_{*}\right),
$$

and then integrate from $t_{3}$ to $t_{*}$ to obtain

$$
\begin{gathered}
u\left(t_{*}\right)>u\left(t_{*}\right)-u\left(t_{3}\right) \geq \int_{t_{3}}^{t_{*}}\left(s-t_{3}\right)(M+N) u\left(t_{*}\right) d s \\
\geq \frac{M+N}{2} u\left(t_{*}\right)\left(t_{*}-t_{3}\right)^{2} \geq \frac{M+N}{2} u\left(t_{*}\right) T^{2}
\end{gathered}
$$

that implies $1<\frac{T^{2}}{2}(M+N)$. This is a contradiction. The proof is complete.

Lemma 2.2 Assume that $u \in E$ satisfies

$$
\left\{\begin{array}{l}
-u^{\prime \prime}(t)+M u(t)+N u(\theta(t)) \\
\quad+\left[\left(M+\frac{\pi^{2}}{T^{2}}\right) c(t)+N c(\theta(t))\right]\left(u^{\prime}(T)-u^{\prime}(0)\right) \leq 0, \quad t \in J, \\
u(0)=u(T), \quad u^{\prime}(0)<u^{\prime}(T) .
\end{array}\right.
$$

where constants $M>0, N \geq 0$ satisfy (2.1). Then $u(t) \leq 0$ for all $t \in J$.

Proof Put

$$
y(t)=u(t)+c(t)\left(u^{\prime}(T)-u^{\prime}(0)\right), \quad t \in J,
$$

then $y \in E$ and $u(t) \leq y(t)$ for all $t \in J$ and

$$
y^{\prime}(t)=u^{\prime}(t)+\frac{1}{2}\left(u^{\prime}(T)-u^{\prime}(0)\right) \cos \frac{\pi}{T} t .
$$

Noting that $y^{\prime \prime}(t)=u^{\prime \prime}(t)-\frac{\pi}{2 T}\left(u^{\prime}(T)-u^{\prime}(0)\right) \sin \frac{\pi}{T} t, t \in J$, we have

$$
\begin{aligned}
& -y^{\prime \prime}(t)+M y(t)+N y(\theta(t)) \\
& =-u^{\prime \prime}(t)+M u(t)+N u(\theta(t))
\end{aligned}
$$




$$
\begin{aligned}
& +\left[\left(M+\frac{\pi^{2}}{T^{2}}\right) c(t)+N c(\theta(t))\right]\left(u^{\prime}(T)-u^{\prime}(0)\right) \leq 0, \\
y(0) & =u(0)=u(T)=y(T), \\
y^{\prime}(0) & =\frac{1}{2}\left(u^{\prime}(T)+u^{\prime}(0)\right)=y^{\prime}(T) .
\end{aligned}
$$

Hence by Lemma 2.1, $y(t) \leq 0$ for all $t \in J$, which implies that $u(t) \leq 0$ for $t \in J$. This completes the proof.

Lemma 2.3 Assume that $u \in E$ satisfies

$$
\left\{\begin{array}{l}
-u^{\prime \prime}(t)+M u(t)+N u(\theta(t)) \leq 0, \quad t \in J, \\
u(0) \leq 0, \quad u(T) \leq 0,
\end{array}\right.
$$

where constants $M>0, N \geq 0$ satisfy (2.1). Then $u(t) \leq 0$ for all $t \in J$.

Proof Suppose, to the contrary, that $u(t)>0$ for some $t \in J$. Then from boundary conditions, we have that there exists a $t^{*} \in(0,1)$ such that

$$
u\left(t^{*}\right)=\max _{t \in J} u(t)>0, \quad u^{\prime}\left(t^{*}\right)=0 .
$$

Suppose that $u(t) \geq 0$ for $t \in J$. It is easy to see that $u(0)=0$ and $u^{\prime \prime}(t) \geq 0$ for $t \in J$. From $u(0)=0$ and $u(t) \geq 0$ for $t \in J$, we obtain that $u^{\prime}(0) \geq 0$. Therefore, $u^{\prime}(t) \geq u^{\prime}(0) \geq 0$. It follows that $u(T)=\max _{t \in J} u(t)>0$, a contradiction.

Suppose that there exist $t_{1}, t_{2} \in J$ such that $u\left(t_{1}\right)>0$ and $u\left(t_{2}\right)<0$. Let $t_{*} \in$ $\left[0, t^{*}\right)$ be such that $u\left(t_{*}\right)=\min _{t \in\left[0, t^{*}\right)} u(t) \leq 0$. From the first inequality of (2.3), we have

$$
u^{\prime \prime}(t) \geq(M+N) u\left(t_{*}\right), \quad t \in\left[0, t^{*}\right) .
$$

Integrating the above inequality from $s\left(t_{*} \leq s \leq t^{*}\right)$ to $t^{*}$, we obtain

$$
-u^{\prime}(s) \geq\left(t^{*}-s\right)(M+N) u\left(t_{*}\right), \quad t_{*} \leq s \leq t^{*},
$$

and then integrate from $t_{*}$ to $t^{*}$ to obtain

$$
\begin{aligned}
-u\left(t_{*}\right) & <u\left(t^{*}\right)-u\left(t_{*}\right) \leq \int_{t_{*}}^{t^{*}}\left(s-t^{*}\right)(M+N) u\left(t_{*}\right) d s \\
& \leq-\frac{M+N}{2} u\left(t_{*}\right)\left(t^{*}-t_{*}\right)^{2} \leq-\frac{M+N}{2} u\left(t_{*}\right) T^{2} .
\end{aligned}
$$

Hence, $u\left(t_{*}\right)\left[2-(M+N) T^{2}\right]>0$, a contradiction. The proof is complete. 


\section{Linear problem}

In this section, we consider the boundary value problem

$$
\left\{\begin{array}{l}
-u^{\prime \prime}(t)+M u(t)+N u(\theta(t))=\sigma(t), \quad t \in J, \\
u(0)=u(T), \quad u^{\prime}(0)=u^{\prime}(T)
\end{array}\right.
$$

where $\sigma \in C(J, R), M, N$ are constants.

Theorem 3.1 Assume that constants $M>0, N \geq 0$ satisfy (2.1) and there exist $\alpha, \beta \in E$ such that

$\left(h_{1}\right) \alpha \leq \beta$ on $J$.

$\left(h_{2}\right)$

$$
\left\{\begin{array}{l}
\left.-\alpha^{\prime \prime}(t)+M \alpha(t)+N \alpha(\theta(t))\right) \leq \sigma(t)-a(t), \quad t \in J \\
\alpha(0)=\alpha(T)
\end{array}\right.
$$

where

$$
a(t)= \begin{cases}0, & \alpha^{\prime}(0) \geq \alpha^{\prime}(T), \\ {\left[\left(M+\frac{\pi^{2}}{T^{2}}\right) c(t)+N c(\theta(t))\right]\left(\alpha^{\prime}(T)-\alpha^{\prime}(0)\right),} & \alpha^{\prime}(0)<\alpha^{\prime}(T) .\end{cases}
$$

$\left(h_{3}\right)$

$$
\left\{\begin{array}{l}
\left.-\beta^{\prime \prime}(t)+M \beta(t)+N \beta(\theta(t))\right) \geq \sigma(t)+b(t), \quad t \in J \\
\beta(0)=\beta(T)
\end{array}\right.
$$

where

$$
b(t)= \begin{cases}0, & \beta^{\prime}(0) \leq \beta^{\prime}(T), \\ {\left[\left(M+\frac{\pi^{2}}{T^{2}}\right) c(t)+N c(\theta(t))\right]\left[\beta^{\prime}(0)-\beta^{\prime}(T)\right],} & \beta^{\prime}(0)>\beta^{\prime}(T) .\end{cases}
$$

Then the boundary value problem (3.1) has a unique solution $u(t)$ and $\alpha \leq u \leq \beta$ for $t \in J$.

Proof We first show that the solution of (3.1) is unique. Let $u_{1}, u_{2}$ be solutions of (3.1) and set $v=u_{1}-u_{2}$. Thus

$$
\left\{\begin{array}{l}
-v^{\prime \prime}(t)+M v(t)+N v(\theta(t))=0, \quad t \in J \\
v(0)=v(T), \quad v^{\prime}(0)=v^{\prime}(T)
\end{array}\right.
$$

By Lemma 2.1, we have that $v \leq 0$ for $t \in J$, that is, $u_{1} \leq u_{2}$ on $J$. Similarly, one can obtain that $u_{2} \leq u_{1}$ on $J$. Hence $u_{1}=u_{2}$.

Next, we prove that if $u$ is a solution of (3.1), then $\alpha \leq u \leq \beta$.

Let $m=\alpha-u$. If $\alpha^{\prime}(0) \geq \alpha^{\prime}(T)$, then $a(t)=0$ on $J$. So we have

$$
\left\{\begin{array}{l}
-m^{\prime \prime}(t)+M m(t)+N m(\theta(t)) \leq 0, \quad t \in J, \\
m(0)=m(T), \quad m^{\prime}(0) \geq m^{\prime}(T) .
\end{array}\right.
$$


By Lemma 2.1, we have that $m=\alpha-u \leq 0$ on $J$.

If $\alpha^{\prime}(0)<\alpha^{\prime}(T)$, then $a(t)=\left[\left(M+\frac{\pi^{2}}{T^{2}}\right) c(t)+N c(\theta(t))\right]\left(\alpha^{\prime}(T)-\alpha^{\prime}(0)\right)$. Thus

$$
\begin{aligned}
- & m^{\prime \prime}(t)+M m(t)+N m(\theta(t)) \\
& \left.=-\alpha^{\prime \prime}(t)+M \alpha(t)+N \alpha(\theta(t))+u^{\prime \prime}(t)-M u(t)-N u(\theta(t))\right) \\
& \leq \sigma(t)-a(t)-\sigma(t)=-a(t) \\
& =-\left[\left(M+\frac{\pi^{2}}{T^{2}}\right) c(t)+N c(\theta(t))\right]\left(m^{\prime}(T)-m^{\prime}(0)\right)
\end{aligned}
$$

It is easy to see that $m(0)=m(T), m^{\prime}(0)<m^{\prime}(T)$. By Lemma 2.2, we have that $m=\alpha-u \leq 0$ on $J$. Analogously, $u \leq \beta$ on $J$.

Finally, we show that (3.1) has a solution by several steps.

Step 1 Consider the equation

$$
\left\{\begin{array}{l}
-u^{\prime \prime}(t)+M u(t)+N u(\theta(t))=\sigma(t), \quad t \in J, \\
u(0)=u(T)=\lambda
\end{array}\right.
$$

where $M, N$ and $\sigma$ are defined in (3.1). For any $\lambda \in R$, we show (3.2) has a unique solution $u(t, \lambda)$.

It is easy to check that (3.2) is equivalent to the integral equation

$$
u(t)=\lambda+\int_{0}^{T} G(t, s)[\sigma(s)-M u(s)-N u(\theta(s))] d s
$$

where

$$
G(t, s)= \begin{cases}\frac{t}{T}(T-s), & 0 \leq t \leq s \leq T, \\ \frac{s}{T}(T-t), & 0 \leq s \leq t \leq T .\end{cases}
$$

Define a mapping $\Phi: C(J, R) \rightarrow C(J, R)$ by

$$
(\Phi u)(t)=\lambda+\int_{0}^{T} G(t, s)[\sigma(s)-M u(s)-N u(\theta(s))] d s .
$$

Obviously $C(J, R)$ is a Banach space with norm $\|u\|=\sup _{t \in J}|u(t)|$. For any $x, y \in$ $C(J, R)$, we have

$$
(\Phi x)(t)-(\Phi y)(t)=\int_{0}^{T} G(t, s)[M(y(s)-x(s))+N(y(\theta(s))-x(\theta(s)))] d s .
$$

The condition (2.1) implies that $\Phi: C(J, R) \rightarrow C(J, R)$ is a contraction mapping since $\max _{t \in J} \int_{0}^{T} G(t, s) d s=T^{2} / 8$. There exists a unique $u \in C(J, R)$ such that $\Phi u=u$. Thus (3.2) has a unique solution $u(t, \lambda)$. Moreover, $u(t, \lambda) \in C^{2}(J, R)$. 
Step 2 We show that for any $t \in J$, the unique solution $u(t, \lambda)$ of the boundary value problem (3.2) is continuous in $\lambda$. Let $u\left(t, \lambda_{i}\right), i=1,2$ be the solution of

$$
\begin{cases}-u^{\prime \prime}(t)+M u(t)+N u(\theta(t))=\sigma(t), & t \in J \\ u(0)=u(T)=\lambda_{i}, & i=1,2\end{cases}
$$

Then

$$
u\left(t, \lambda_{i}\right)=\lambda_{i}+\int_{0}^{T} G(t, s)\left[\sigma(s)-M u\left(s, \lambda_{i}\right)-N u\left(\theta(s), \lambda_{i}\right)\right] d s, \quad i=1,2 .
$$

From (3.5), we have that

$$
\begin{aligned}
& \left\|u\left(t, \lambda_{1}\right)-u\left(t, \lambda_{2}\right)\right\| \\
& \quad \leq\left|\lambda_{1}-\lambda_{2}\right|+(M+N)\left\|u\left(t, \lambda_{1}\right)-u\left(t, \lambda_{2}\right)\right\| \max _{t \in J} \int_{0}^{T} G(t, s) d s \\
& \quad \leq\left|\lambda_{1}-\lambda_{2}\right|+\frac{T^{2}}{8}(M+N)\left\|u\left(t, \lambda_{1}\right)-u\left(t, \lambda_{2}\right)\right\| .
\end{aligned}
$$

Hence

$$
\left\|u\left(t, \lambda_{1}\right)-u\left(t, \lambda_{2}\right)\right\| \leq \frac{8}{8-T^{2}(M+N)}\left|\lambda_{1}-\lambda_{2}\right| .
$$

Step 3 We show that for any $t \in J, u_{t}(t, \lambda)$ is continuous in $\lambda$, where $u(t, \lambda)$ is unique solution of the problem (3.2).

Since $u(t, \lambda) \in C^{2}(J, R), u_{t}(t, \lambda)$ exists for any $t \in J$ and $\lambda \in R$. From (3.5) and (3.6), we have

$$
\begin{aligned}
& \left\|u_{t}\left(t, \lambda_{1}\right)-u_{t}\left(t, \lambda_{2}\right)\right\| \\
& \quad \leq \frac{M+N}{T}\left\|u\left(t, \lambda_{1}\right)-u\left(t, \lambda_{2}\right)\right\| \max _{t \in J}\left(\int_{0}^{t} s d s+\int_{t}^{T}(T-s) d s\right) \\
& \quad \leq \frac{(M+N) T}{2}\left\|u\left(t, \lambda_{1}\right)-u\left(t, \lambda_{2}\right)\right\| \\
& \quad \leq \frac{4(M+N) T}{8-T^{2}(M+N)}\left|\lambda_{1}-\lambda_{2}\right| .
\end{aligned}
$$

Step 4 We show that there exists one $\lambda$ such that $u_{t}(0, \lambda)=u_{t}(T, \lambda)$, where $u(t, \lambda)$ is the unique solution of the problem (3.2).

Put

$$
\begin{aligned}
& \bar{\alpha}(t)= \begin{cases}\alpha(t), & \alpha^{\prime}(0) \geq \alpha^{\prime}(T), \\
\alpha(t)+c(t)\left(\alpha^{\prime}(T)-\alpha^{\prime}(0)\right), & \alpha^{\prime}(0)<\alpha^{\prime}(T),\end{cases} \\
& \bar{\beta}(t)= \begin{cases}\beta(t), & \beta^{\prime}(0) \leq \beta^{\prime}(T), \\
\beta(t)-c(t)\left(\beta^{\prime}(0)-\beta^{\prime}(T)\right), & \beta^{\prime}(0)>\beta^{\prime}(T),\end{cases}
\end{aligned}
$$


then $\alpha(t) \leq \bar{\alpha}(t), \bar{\beta}(t) \leq \beta(t)$ for any $t \in J$ and

$$
\begin{aligned}
& \left\{\begin{array}{l}
-\bar{\alpha}^{\prime \prime}(t)+M \bar{\alpha}(t)+N \bar{\alpha}(\theta(t)) \leq \delta(t), \quad t \in J, \\
\bar{\alpha}(0)=\bar{\alpha}(T), \quad \bar{\alpha}^{\prime}(0) \geq \bar{\alpha}^{\prime}(T),
\end{array}\right. \\
& \left\{\begin{array}{l}
-\bar{\beta}^{\prime \prime}(t)+M \bar{\beta}(t)+N \bar{\beta}(\theta(t)) \geq \delta(t), \quad t \in J, \\
\bar{\beta}(0)=\bar{\beta}(T), \quad \bar{\beta}^{\prime}(0) \leq \bar{\beta}^{\prime}(T) .
\end{array}\right.
\end{aligned}
$$

Using (3.7) and (3.8), one easily obtain that $\bar{\alpha}(t) \leq \bar{\beta}(t)$ for any $t \in J$.

Put $\lambda \in[\bar{\alpha}(0), \bar{\beta}(0)]$, then $\bar{\alpha}(0)=\bar{\alpha}(T) \leq u(0, \lambda)=u(T, \lambda) \leq \bar{\beta}(0)=\bar{\beta}(T)$. Using Lemma 2.3 , one easily obtain that $\bar{\alpha}(t) \leq u(t, \lambda) \leq \bar{\beta}(t)$ on $J$. Hence

$$
\begin{array}{ll}
u_{t}(0, \bar{\alpha}(0)) \geq \bar{\alpha}^{\prime}(0), & u_{t}(T, \bar{\alpha}(T)) \leq \bar{\alpha}^{\prime}(T), \\
u_{t}(0, \bar{\beta}(0)) \leq \bar{\beta}^{\prime}(0), & u_{t}(T, \bar{\beta}(T)) \geq \bar{\beta}^{\prime}(T) .
\end{array}
$$

Define a function

$$
P(\lambda)=u_{t}(0, \lambda)-u_{t}(T, \lambda),
$$

where $u(t, \lambda)$ is the unique solution of the problem (3.2). Since $P$ is continuous and

$$
P(\bar{\alpha}(0)) P(\bar{\beta}(0)) \leq 0,
$$

there exists one $\lambda_{0} \in[\bar{\alpha}(0), \bar{\beta}(0)]$ such that $P\left(\lambda_{0}\right)=0$, that is, $u_{t}\left(0, \lambda_{0}\right)=u_{t}\left(T, \lambda_{0}\right)$. Obviously, $u\left(t, \lambda_{0}\right)$ is unique solution of the problem (3.1). This completes the proof.

Remark 2 We remove the condition $\min \{M, \sqrt{M}\}>N$ in [6].

\section{Main result}

Let $M \in R, N \in R$. We first give the following definition.

Definition 4.1 A function $\alpha \in E$ is called a lower solution of the boundary value problem (1.1) if

$$
\left\{\begin{array}{l}
-\alpha^{\prime \prime}(t) \leq f(t, \alpha(t), \alpha(\theta(t)))-a(t), \quad t \in J, \\
\alpha(0)=\alpha(T)
\end{array}\right.
$$

where

$$
a(t)= \begin{cases}0, & \alpha^{\prime}(0) \geq \alpha^{\prime}(T), \\ {\left[\left(M+\frac{\pi^{2}}{T^{2}}\right) c(t)+N c(\theta(t))\right]\left(\alpha^{\prime}(T)-\alpha^{\prime}(0)\right),} & \alpha^{\prime}(0)<\alpha^{\prime}(T) .\end{cases}
$$


Definition 4.2 A function $\beta \in E$ is called an upper solution of the boundary value problem (1.1) if

$$
\left\{\begin{array}{l}
-\beta^{\prime \prime}(t) \geq f(t, \beta(t), \beta(\theta(t)))+b(t), \quad t \in J \\
\beta(0)=\beta(T)
\end{array}\right.
$$

where

$$
b(t)= \begin{cases}0, & \beta^{\prime}(0) \leq \beta^{\prime}(T), \\ {\left[\left(M+\frac{\pi^{2}}{T^{2}}\right) c(t)+N c(\theta(t))\right]\left[\beta^{\prime}(0)-\beta^{\prime}(T)\right],} & \beta^{\prime}(0)>\beta^{\prime}(T) .\end{cases}
$$

Theorem 4.1 Assume that the following conditions are satisfied

$\left(H_{1}\right)$ The functions $\alpha, \beta$ are lower and upper solutions of (1.1) respectively, and $\alpha \leq \beta$ on $J$.

$\left(H_{2}\right)$ The constants $M, N$ in definition of upper and lower solutions satisfy (2.1) and

$$
f(t, x, y)-f(t, \bar{x}, \bar{y}) \geq-M(x-\bar{x})-N(y-\bar{y})
$$

for $\alpha(t) \leq \bar{x} \leq x \leq \beta(t), \alpha(\theta(t)) \leq \bar{y} \leq y \leq \beta(\theta(t)), t \in J$.

Then, there exist monotone sequences $\left\{\alpha_{n}\right\},\left\{\beta_{n}\right\}$ with $\alpha_{0}=\alpha, \beta_{0}=\beta$ such that $\lim _{n \rightarrow \infty} \alpha_{n}(t)=\rho(t), \lim _{n \rightarrow \infty} \beta_{n}(t)=r(t)$ uniformly on $J$, and $\rho, r$ are the minimal and the maximal solutions of (1.1) respectively, such that

$$
\alpha_{0} \leq \alpha_{1} \leq \alpha_{2} \leq \cdots \leq \alpha_{n} \leq \rho \leq x \leq r \leq \beta_{n} \leq \cdots \leq \beta_{2} \leq \beta_{1} \leq \beta_{0}
$$

on $J$, where $x$ is any solution of (1.1) such that $\alpha(t) \leq x(t) \leq \beta(t)$ on $J$.

Proof Let $[\alpha, \beta]=\{u \in E: \alpha(t) \leq u(t) \leq \beta(t), t \in J\}$. For any $\gamma \in[\alpha, \beta]$, we consider the equation

$$
\left\{\begin{array}{l}
-u^{\prime \prime}(t)+M u(t)+N u(\theta(t))=f(t, \gamma(t), \gamma(\theta(t)))+M \gamma(t)+N \gamma(\theta(t)), \\
\quad t \in J, \\
u(0)=u(T), \quad u^{\prime}(0)=u^{\prime}(T) .
\end{array}\right.
$$

Theorem 3.1 implies that the problem (4.1) has a unique solution $u \in E$. We define an operator $A$ by $u=A \gamma$, then $A$ is an operator from $[\alpha, \beta]$ to $[\alpha, \beta]$.

We shall show that

(a) $\alpha \leq A \alpha, A \beta \leq \beta$.

(b) $A$ is nondecreasing in $[\alpha, \beta]$.

From $A \alpha \in[\alpha, \beta]$ and $A \beta \in[\alpha, \beta]$, we have that (a) holds. To prove (b). We show that $A \mu_{1} \leq A \mu_{2}$ if $\alpha \leq \mu_{1} \leq \mu_{2} \leq \beta$.

Let $\rho_{1}^{*}=A \mu_{1}, \rho_{2}^{*}=A \mu_{2}$ and $p=\rho_{1}^{*}-\rho_{2}^{*}$, then by $\left(H_{2}\right)$, we have

$$
\begin{aligned}
- & \left.p^{\prime \prime}(t)+M p(t)+N p(\theta(t))\right) \\
= & f\left(t, \mu_{1}(t), \mu_{1}(\theta(t))\right)+M \mu_{1}(t)+N \mu_{1}(\theta(t)) \\
& \quad-f\left(t, \mu_{2}(t), \mu_{2}(\theta(t))\right)-M \mu_{2}(t)-N \mu_{2}(\theta(t)) \leq 0,
\end{aligned}
$$


and $p(0)=p(T), \quad p^{\prime}(0)=p^{\prime}(T)$. By Lemma 2.1, $p(t) \leq 0$, which implies $A \mu_{1} \leq$ $A \mu_{2}$.

Let $\alpha_{n+1}=A \alpha_{n}, \beta_{n+1}=A \beta_{n}$ with $\alpha_{0}=\alpha, \beta_{0}=\beta$. From (a) and (b), we have

$$
\alpha_{0} \leq \alpha_{1} \leq \alpha_{2} \leq \cdots \leq \alpha_{n} \leq \beta_{n} \leq \cdots \leq \beta_{2} \leq \beta_{1} \leq \beta_{0}
$$

on $t \in J$, and each $\alpha_{n}, \beta_{n} \in E$ satisfy

$$
\begin{aligned}
& \left\{\begin{array}{l}
-\alpha_{n}^{\prime \prime}(t)+M \alpha_{n}(t)+N \alpha_{n}(\theta(t)) \\
=f\left(t, \alpha_{n-1}(t), \alpha_{n-1}(\theta(t))\right)+M \alpha_{n-1}(t)+N \alpha_{n-1}(\theta(t)), \quad t \in J, \\
\alpha_{n}(0)=\alpha_{n}(T), \quad \alpha_{n}^{\prime}(0)=\alpha_{n}^{\prime}(T) .
\end{array}\right. \\
& \begin{cases}-\beta_{n}^{\prime \prime}(t)+M \beta_{n}(t)+N \beta_{n}(\theta(t)) & \\
=f\left(t, \beta_{n-1}(t), \beta_{n-1}(\theta(t))\right)+M \beta_{n-1}(t)+N \beta_{n-1}(\theta(t)), \quad t \in J, & \\
\beta_{n}(0)=\beta_{n}(T), \quad \beta_{n}^{\prime}(0)=\beta_{n}^{\prime}(T) . & \end{cases}
\end{aligned}
$$

Therefore there exist $\rho, r$ such that $\lim _{n \rightarrow \infty} \alpha_{n}(t)=\rho(t), \lim _{n \rightarrow \infty} \beta_{n}(t)=r(t)$ uniformly on $J$. Clearly, $\rho, r$ are solutions of (1.1).

Finally, we prove that if $x \in\left[\alpha_{0}, \beta_{0}\right]$ is one solution of (1.1), then $\rho(t) \leq x(t) \leq$ $r(t)$ on $J$. To this end, we assume, without loss of generality, that $\alpha_{n}(t) \leq x(t) \leq$ $\beta_{n}(t)$ for some $n$. From property (b), we can get that

$$
\alpha_{n+1}(t) \leq x(t) \leq \beta_{n+1}(t), \quad t \in J
$$

Since $\alpha_{0}(t) \leq x(t) \leq \beta_{0}(t)$, we can conclude that

$$
\alpha_{n}(t) \leq x(t) \leq \beta_{n}(t), \quad \text { for all } n
$$

Passing to the limit as $n \rightarrow \infty$, we obtain $\rho(t) \leq x(t) \leq r(t), t \in J$. This completes the proof.

Example 1 Consider the equation

$$
\left\{\begin{array}{l}
-u^{\prime \prime}(t)=u\left(\frac{t}{2}\right)-u^{2}(t)+t \sin t, \quad 0 \leq t \leq 0.5 \\
u(0)=u(0.5), \quad u^{\prime}(0)=u^{\prime}(0.5)
\end{array}\right.
$$

Clearly, $\alpha=1$ and $\beta=2$ are lower and upper solutions of (4.2) respectively. Let $M=4, N=1$, then the conditions of Theorem 4.1 are satisfied. Hence, (4.2) has maximal and minimal solutions on $[1,2]$.

Example 2 Consider the equation

$$
\left\{\begin{array}{l}
-u^{\prime \prime}(t)=-\exp \left(-\frac{u(\sin t)+u(t)}{2 A}\right)+\frac{A-u(t)}{A} \int_{0}^{u(t)} e^{-\frac{r^{2}}{16}} d r, \quad 0 \leq t \leq \pi, \\
u(0)=u(\pi), \quad u^{\prime}(0)=u^{\prime}(\pi),
\end{array}\right.
$$


where $A>\max \left\{10^{5}, B\right\}$ with $B>0$ and $\int_{0}^{B-1} e^{-\frac{r^{2}}{16}} d r \geq \frac{3}{2} \sqrt{\pi}$ (since $\int_{0}^{\infty} e^{-\frac{r^{2}}{16}} d r=$ $2 \sqrt{\pi}$, such a $B$ exists). Setting

$$
\begin{aligned}
& \alpha=A-1, \quad \beta=A-\frac{t(t-\pi)}{100} \\
& f(t, x, y)=-\exp \left(-\frac{x+y}{2 A}\right)+\frac{A-x}{A} \int_{0}^{x} e^{-\frac{r^{2}}{16}} d r .
\end{aligned}
$$

It is easy to check that $\alpha$ is one lower solution of (4.3). We show that $\beta$ is one upper solution of (4.3). Noting that

$$
\begin{aligned}
& f_{x}(t, x, y)=\frac{1}{2 A} \exp \left(-\frac{x+y}{2 A}\right)-\frac{1}{A} \int_{0}^{x} e^{-\frac{r^{2}}{16}} d r+\frac{A-x}{A} e^{-\frac{x^{2}}{16}} \geq-10^{-4}, \\
& f_{y}(t, x, y)=\frac{1}{2 A} \exp \left(-\frac{x+y}{2 A}\right)>0
\end{aligned}
$$

for $x, y \in[\alpha, \beta] \subset[A-1, A+1]$, we put $M=N=0.001$, then the condition $\left(H_{2}\right)$ of Theorem 4.1 is satisfied. On the other hand, $\beta^{\prime}(0)-\beta^{\prime}(T)=\pi / 50$,

$$
\begin{gathered}
{\left[\left(M+\frac{\pi^{2}}{T^{2}}\right) c(t)+N c(\theta(t))\right]\left[\beta^{\prime}(0)-\beta^{\prime}(T)\right] \leq \frac{\pi+1}{100},} \\
f(t, \beta(t), \beta(\sin t)) \leq-e^{-1}+\frac{3 t(t-\pi) \sqrt{\pi}}{200 A}, \\
-\beta^{\prime \prime}=\frac{2}{100}>-e^{-1}+\frac{3 t(t-\pi) \sqrt{\pi}}{200 A}+\frac{\pi+1}{100} \\
>f(t, \beta(t), \beta(\sin t))+\left[\left(M+\frac{\pi^{2}}{T^{2}}\right) c(t)+N c(\theta(t))\right]\left[\beta^{\prime}(0)-\beta^{\prime}(T)\right] .
\end{gathered}
$$

The conditions of Theorem 4.1 are satisfied. Hence, (4.3) has maximal and minimal solutions on $[\alpha, \beta]$.

Acknowledgements The authors thank the referee for his/her careful reading and useful comments. This work is supported by Scientific Research Fund of Hunan Provincial Education Department (09B033) and by Hunan Provincial Natural Science Foundation of China (09JJ3010).

Open Access This article is distributed under the terms of the Creative Commons Attribution Noncommercial License which permits any noncommercial use, distribution, and reproduction in any medium, provided the original author(s) and source are credited.

\section{References}

1. Ladde, G.S., Lakshmikantham, V., Vatsala, A.S.: Monotone Iterative Techniques for Nonlinear Differential Equations. Pitman Advanced Publishing Program. Pitman, London (1985)

2. Nieto, J.J., Rodriguez-Lopez, R.: Existence and approximation of solutions for nonlinear function differential equations with periodic boundary value conditions. J. Comput. Appl. Math. 40, 433-442 (2000) 
3. Nieto, J.J., Rodriguez-Lopez, R.: Remark on periodic boundary value problems for function differential equations. J. Comput. Appl. Math. 158, 339-353 (2003)

4. He, Z., He, X.: Periodic boundary value problems for first order impulsive integro-differential equations of mixed type. J. Math. Anal. Appl. 296, 8-20 (2004)

5. Ding, W., Mi, J.R., Han, M.: Periodic boundary value problems for the first order impulsive functional differential equations. Appl. Math. Comput. 165, 443-456 (2005)

6. Ding, W., Han, M., Yan, J.: Periodic boundary value problems for the second order functional equations. J. Math. Anal. Appl. 298, 341-351 (2004)

7. Henderson, J.: Boundary Value Problems for Functional Equations. World Scientific, Singapore (1995)

8. Haddock, J.R., Nkashama, M.N.: Periodic boundary value problems and monotone iterative methods for functional differential equations. Nonlinear Anal. 22(3), 267-276 (1994)

9. Jankowski, T.: Existence of solutions of boundary value problems for differential equations with delayed arguments. J. Comput. Appl. Math. 156, 239-252 (2003)

10. Jankowski, T.: Advanced differential equations with nonlinear boundary conditions. J. Math. Anal. Appl. 304, 490-503 (2005)

11. Jankowski, T.: Solvability of three point boundary value problems for second order ordinary differential equations with deviating arguments. J. Math. Anal. Appl. 312, 620-636 (2005)

12. Nieto, J.J.: Periodic boundary value problems for first-order impulsive ordinary differential equations. Nonlinear Anal. TMA 51(2), 1223-1232 (2002)

13. Nieto, J.J., Cabada, A.: A generalized upper and lower solution method for nonlinear second order ordinary differential equations. J. Appl. Math. Stoch. Anal. 5, 157-165 (1992)

14. Nieto, J.J., Rodriguez-Lopez, R.: Monotone method for first-order functional differential equations. Comput. Math. Appl. 52, 471-484 (2006)

15. Wang, W., Yang, X., Shen, J.: Boundary value problems involving upper and lower solutions in reverse order. J. Comput. Appl. Math. 230, 1-7 (2009)

16. Zuo, W., Jiang, D., O’Regan, D., Agarwal, R.P.: A monotone method for fourth order periodic boundary value problems and periodic solutions of functional differential equations. Methods Appl. Anal 12, 19-28 (2005) 